Behavioral/Systems/Cognitive

\title{
The Neural Correlates of Visual and Verbal Cognitive Styles
}

\author{
David J. M. Kraemer, Lauren M. Rosenberg, and Sharon L. Thompson-Schill \\ Center for Cognitive Neuroscience, Department of Psychology, University of Pennsylvania, Philadelphia, Pennsylvania 19104-6241
}

\begin{abstract}
It has long been thought that propensities for visual or verbal learning styles influence how children acquire knowledge successfully and how adults reason in everyday life. There is no direct evidence to date, however, linking these cognitive styles to specific neural systems. In the present study, visual and verbal cognitive styles are measured by self-report survey, and cognitive abilities are measured by scored tests of visual and verbal skills. Specifically, we administered the Verbalizer-Visualizer Questionnaire (VVQ) and modality-specific subtests of the Wechsler Adult Intelligence Scale (WAIS) to 18 subjects who subsequently participated in a functional magnetic resonance imaging experiment. During the imaging session, participants performed a novel psychological task involving both word-based and picture-based feature matching conditions that was designed to permit the use of either a visual or a verbal processing style during all conditions of the task. Results demonstrated a pattern of activity in modality-specific cortex that distinguished visual from verbal cognitive styles. During the word-based condition, activity in a functionally defined brain region that responded to viewing pictorial stimuli (fusiform gyrus) correlated with self-reported visualizer ratings on the VVQ. In contrast, activity in a phonologically related brain region (supramarginal gyrus) correlated with the verbalizer dimension of the VVQ during the picture-based condition. Scores from the WAIS subtests did not reliably correlate with brain activity in either of these regions. These findings suggest that modality-specific cortical activity underlies processing in visual and verbal cognitive styles.
\end{abstract}

\section{Introduction}

Some individuals prefer learning through pictures and others through words. This basic notion, familiar to the fields of Psychology and Education, has inspired and been furthered by theories about visual and verbal cognitive styles and the related topic of multiple intelligences (Spearman, 1927; Guilford, 1967; Gardner, 1993; Mayer and Massa, 2003).

The original method of identifying propensities for verbal or visual cognition, the "Verbalizer-Visualizer Questionnaire" (VVQ) (Richardson, 1977), had several shortcomings (Kirby et al., 1988; Green and Schroeder, 1990; Boswell and Pickett, 1991; Blajenkova et al., 2006). However, a revision was shown to have greater construct validity (Kirby et al., 1988). Although behavioral evidence supports the link between these cognitive styles and certain cognitive abilities (Kirby et al., 1988; Mayer and Massa, 2003), little is currently known about the neural basis of these differences.

There is, however, an extensive neuroimaging literature on processing modality-specific information. Many brain regions involved in visual perception are also involved in visually based semantic knowledge, working memory, and imagery (Martin, 2000; Kosslyn and Thompson-Schill, 2003; Postle et al., 2003; Ranganath and D'Esposito, 2005; Goldberg et al., 2006; Cui et al., 2007). Likewise, left perisylvian regions involved in phonological

\footnotetext{
Received Sept. 26, 2008; revised Feb. 9, 2009; accepted Feb. 11, 2009.

This work was supported by National Institutes of Health Grant R01 MH70850 to S.L.T.-S. We thank D. Hackman for advice and guidance regarding administration of the WAIS.

Correspondence should be addressed to David J. M. Kraemer, Center for Cognitive Neuroscience, Department of Psychology, University of Pennsylvania, 3810 Walnut Street, Philadelphia, PA 19104-6241. E-mail: dkraemer@psych.upenn.edu.

D0I:10.1523/JNEUROSCI.4635-08.2009

Copyright $\odot 2009$ Society for Neuroscience $\quad$ 0270-6474/09/293792-07\$15.00/0
}

and speech processing are also involved in verbal working memory and auditory imagery (Paulesu et al., 1993; Smith and Jonides, 1998; Halpern and Zatorre, 1999; Kraemer et al., 2005). Consequently, we hypothesize that visual brain regions subserve the visual cognitive style and that the verbal cognitive style relies on brain areas linked to phonology and verbal working memory.

In the present study, we explore this hypothesis using functional magnetic resonance imaging (fMRI). Our task (see Fig. 1) used a target-probe design in which stimuli could appear either as images or words, producing four within-subject experimental conditions: picture-picture, word-word, picture-word, and word-picture. The task had three important properties: (1) subjects could complete the task using either a verbal or a visual strategy on single-modality (picture-picture/word-word) trials; (2) subjects had an incentive to "convert" from their nonpreferred modality to their preferred modality, if they had one, because of the inclusion of an equal number of cross-modal (picture-word/word-picture) trials; (3) performance was comparable across both single-modality conditions, regardless of cognitive style (i.e., the task minimized performance differences that might otherwise confound fMRI comparisons).

We focused our analyses on the single-modality conditions, using VVQ scores as predictors of task-related brain activity and the working memory literature to identify regions of interest. We predicted that (1) during the word-word condition, a propensity for the visual style would correlate with activity in visual working memory regions; and (2) during the picture-picture condition, a propensity for the verbal style would correlate with activity in verbal working memory regions. Such a pattern of results would indicate that cognitive styles are associated with processing information in one's preferred modality, even when it is presented in a nonpreferred form (e.g., converting visual information into a 
verbal working memory representation). Thus, the present study provides a useful first description of the biological basis of these individual differences in cognition.

\section{Materials and Methods}

Participants. Eighteen right-handed individuals [11 women; mean $(\mathrm{M})=22.2$ years] with normal or corrected-to-normal vision participated in this study. Strong right-handedness was verified with a modified form of the Annett Handedness Questionnaire (Annett, 1970). All participants were native speakers of English and had not learned any other language before age five. No participants indicated any learning disabilities or any history of neurological or psychiatric disorders. Participants were recruited from local universities, through the Center for Cognitive Neuroscience at the University of Pennsylvania. In accordance with the Institutional Review Board at the University of Pennsylvania, all participants provided informed consent and were monetarily compensated for participation.

Measure of self-reported verbal and visual cognitive style. A computerized version of the revised Verbalizer-Visualizer Questionnaire (Kirby et al., 1988) was administered to assess the degree to which participants employ visual and verbal reasoning in common tasks and situations. The VVQ (following modifications developed by Kirby et al., 1988) consists of 10 statements that relate to a verbal reasoning style and 10 that relate to a visual reasoning style (10 additional questions assess a third "dream vividness" dimension which was not included in later analysis). Participants rated each statement on a discrete five-point scale from strongly agree (5) to strongly disagree (1). Scores for the visual and verbal statements were calculated separately, where we summed the responses of all 10 questions within that dimension. The five positively worded questions received positive scores, whereas the five negatively worded questions were given negative scores, thus creating a range of possible scores from -20 to 20 . The questionnaire was presented on a computer using E-Prime software (Psychology Software Tools), with text appearing in black over a white background.

Objective tests of visual and verbal cognitive abilities. Subtests of the Wechsler Adult Intelligence Scale (WAIS), Third Edition (Wechsler, 1997), were used to assess visual and verbal reasoning ability. We administered six subtests consecutively: picture completion, vocabulary, similarities, block design, matrix reasoning, and information. Verbal Comprehension Index (VCI) scores were calculated from performance on vocabulary, similarities, and information. Perceptual Organization Index (POI) scores were calculated from performance on picture completion, block design, and matrix reasoning.

Similarity judgment task. Participants completed a similarity judgment task while undergoing fMRI. As shown in Figure 1, each item in this task consisted of either an image (e.g., a red triangle with stripes) or a set of three words that named a shape, color, and pattern (e.g., red, stripes, triangle). There were five possible attribute assignments to each of the three features that composed each item.

On each experimental trial, participants first viewed a target item (either picture or words) in the center of the screen for $1500 \mathrm{~ms}$. Subsequently, two probe items (either two pictures or two sets of words) appeared for $3500 \mathrm{~ms}$ before disappearing. As targets and probes could be presented either as images or as words, there were four within-subject trial conditions: picture-picture, word-word, picture-word, and wordpicture. Participants completed 30 trials of each of these four conditions. No three-item set (target and two probes) was ever repeated within or across condition type.

When the probe items appeared, participants indicated via button press which of the two probes was more similar to the previous target. Participants had $3500 \mathrm{~ms}$ to respond before the probes disappeared. The probes were then immediately replaced by a central fixation cross for $1 \mathrm{~s}$. In half of the experimental trials, the correct answer was the left probe, and in the other half of the experimental trials, the correct answer was the right probe. Correct probes contained two of the three features in common with the target; incorrect items only contained one feature in common. This rule was not explicitly stated to participants; instead, feedback was given on several practice trials, and all participants demonstrated

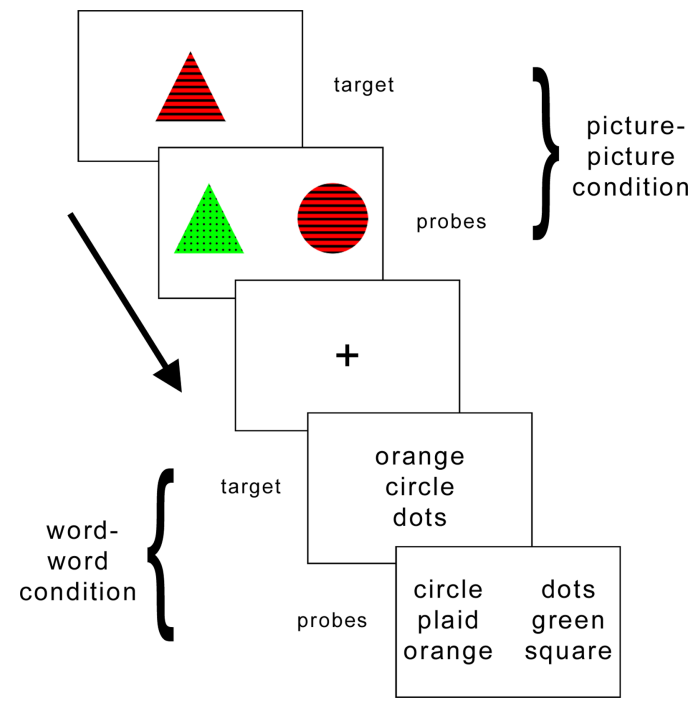

Figure 1. Sample trials of two experimental conditions in the event-related fMRI task design. Targets appeared for $1500 \mathrm{~ms}$, probes for $3500 \mathrm{~ms}$. Fixation trials appeared between experimental trials in a pseudo-random manner such that the time between trials was variable, but no experimental trial began $<1 \mathrm{~s}$ after the previous one ended.

proficiency at the task before the start of the first run. Pilot data indicated that the stimuli depicted as images had high name agreement with the associated verbal labels.

Data collection. Participation involved two experimental sessions. No more than 2 weeks elapsed between the two sessions. In the first session, participants provided informed consent and then completed the WAIS subtests, followed by a Vividness of Visual Imagery Questionnaire (Marks, 1973), and finally the VVQ. Administration of this session lasted $\sim 1 \mathrm{~h}$.

In the second session, participants completed the similarity judgment task while undergoing fMRI scanning. After the completion of the practice trials and an anatomical scan, the participant completed the experimental task. In total, this session lasted $\sim 40 \mathrm{~min}$. Because of equipment failure, data were not collected during functional scanning for one run of one of the participants. For all other participants, three full functional runs were collected. See supplemental material, available at www. jneurosci.org, for more details about fMRI data collection and analysis.

An event-related stochastic design was implemented wherein all four experimental conditions (picture-picture, word-word, picture-word, word-picture), as well as jittered $6 \mathrm{~s}$ fixation periods, were intermixed in a pseudorandom manner into three runs of $8 \mathrm{~min}$ each. Ten trials of each of the experimental conditions appeared in each run, and twenty $6 \mathrm{~s}$ fixation trials were also included in each run.

Each of the 60 fixation trials lasted $6 \mathrm{~s}$ and were otherwise identical to the $1 \mathrm{~s}$ fixation that appeared at the end of every experimental trial. During the analysis, the $6 \mathrm{~s}$ fixation trials were split into two conditions; one half of the fixation trials were used as a baseline (left unmodeled) and the other half were used as a control condition. This was done so that we could assess brain activity during a set of fixation periods as it tracked with our parametric predictor variables (VVQ scores). As the $6 \mathrm{~s}$ fixation trials were pseudorandomly intermixed between other trials (and sometimes appeared in succession), we were able to limit the amount of covariance between fixation trials used for baseline, fixation trials used for the parametric analysis, and experimental trials (Friston et al., 1999).

Two other tasks were also performed in the same runs and they were originally intended as potential additional control conditions. These consisted of two tasks in which participants merely pressed a button at the onset of visual stimulus. One type of stimulus was an abstract grouping of color patches, using the same colors as in the picture condition. The other type of stimulus consisted of pronounceable pseudowords, which the subjects were also instructed to read to themselves during viewing. These tasks are not discussed further as they were unnecessary for the present analyses. 
Preceding the first run, eight experimental trials, composed of two trials from each of the four experimental conditions, were provided to the participant with feedback to ensure that each participant understood the task instructions. No feedback was given during the scanning session.

\section{Results}

\section{Behavioral results}

Each dimension of the VVQ has a possible range of -20 to 20 . Scores on both dimensions of the VVQ were generally positive, although a wide range was obtained for both the verbal dimension (range, -6 to 16 ; $\mathrm{M}=5.2$ ) and the visual dimension (range, $0-19 ; \mathrm{M}=10.6$ ). WAIS: POI scores ranged from 101 (53rd percentile) to 133 (99th percentile), with a group mean score of 118.1. WAIS: VCI scores ranged from 103 (58th percentile) to 140 (99th percentile), with a group mean score of 122.8. Thus, as an expected result of the selection procedure (recruitment from the undergraduate and graduate student populations of the University of Pennsylvania and nearby universities), the present cohort of participants comprised individuals with above-average intelligence, as measured by these two indices of the WAIS.

Table 1 reports Pearson's correlation values $(r)$ for the correlations between each WAIS and VVQ measure as well as difference scores within each separate measure. Based on previous work mentioned above (Kirby et al., 1988) as well as pilot testing in our own laboratory, the verbal and visual dimensions of the VVQ were expected to correlate significantly with the VCI and POI scores from the WAIS, respectively. As reported in Table 1, this was not the case within the present group of participants with respect to the separate visual and verbal scales. However, the difference scores calculated by subtracting the verbal from the visual scores on each measure (i.e., POI-VCI for the WAIS, and Visual Dimension-Verbal Dimension for the VVQ) were significantly correlated $\left(r_{(17)}=0.63, p=0.005\right)$. This indicates that there was similarity in the classification of visual and verbal propensities between the tests of ability (WAIS) and preference (VVQ), although the individual dimensions were not significantly correlated in the present sample. As this lack of a significant correlation between our separate measures of cognitive ability (POI and VCI) and the visualizer and verbalizer scores of the VVQ is inconsistent with previous findings (Kirby et al., 1988; Mayer and Massa, 2003) and we do not have the statistical power in the present experiment to interpret this null finding, it will not be discussed further here.

As expected, mean response times (ms) varied by experimental condition because of the difference in difficulty between congruent and incongruent conditions [correct trials: word-word: $\mathrm{M}=1674.66, \mathrm{SD}=406.22$; picture-picture: $\mathrm{M}=983.49, \mathrm{SD}=$ 272.24; word-pictures: $\mathrm{M}=996.52, \mathrm{SD}=180.10$; picture-word: $\mathrm{M}=1768.20, \mathrm{SD}=367.33$ ]. Neither VVQ dimension (verbalizer or visualizer) was significantly correlated with response time for any condition. Likewise, neither WAIS index (VCI or POI) was significantly correlated with response time for any condition. Average accuracy was high for the main conditions of interest (word-word, 87.96\%; picture-picture, 92.40\%). Accuracy was lower for the more difficult incongruent conditions (word-picture, 54.07\%; picture-word, 77.96\%). Neither VVQ dimension was significantly correlated with accuracy for any condition. Likewise, neither WAIS index was significantly correlated with accuracy for any condition.
Table 2. Pearson's correlations ( $r$ ) between VVQ scores and left supramarginal ROI activity by task

\begin{tabular}{lcl}
\hline & VVQ: visualizer & VVQ: verbalizer \\
\hline Picture-picture condition & 0.20 & $0.63^{* *}$ \\
Word-word condition & 0.23 & 0.32 \\
Fixation condition & -0.15 & 0.43 \\
\hline
\end{tabular}

${ }^{* *} p<0.01 ; p$ values calculated for two-tailed tests.

\section{Definition of functional regions of interest}

To establish functionally defined regions of interest (ROIs) that were responsive across subjects as a function of task condition, we performed a random effects analysis over the entire participant group comparing brain activity for the word-word condition versus the picture-picture condition. These whole-brain analyses were assessed at an uncorrected voxel threshold of $t_{(17)}>3.60$, $p<0.001$ (see figure in supplemental material, available at www. jneurosci.org). As expected, the picture-picture condition demonstrated increased activation relative to the word-word condition in bilateral fusiform gyrus, in addition to other brain regions. Also as expected, the reverse contrast (word-word $>$ picturepicture) demonstrated increased activation in left perisylvian cortex, in particular the supramarginal gyrus (SMG), as well as other brain areas. More detailed results from this analysis are presented in supplemental material, available at www.jneurosci. org, including complete maps of the main effects contrasts.

We then generated ROI masks from these contrasts as follows. For our ROI selection criteria, we were focused on brain regions previously implicated in verbal and visual working memory, as we hypothesized these to be the underlying mechanisms relevant to verbal and visual cognitive styles. Based on prominent reviews of the neuroimaging literature written in the past 5 years (Baddeley, 2003; Wager and Smith, 2003; Postle, 2006; D'Esposito, 2007), these regions include bilateral midanterior fusiform gyrus (BA 19/37; visual), left inferior frontal gyrus (BA 44; verbal), left supramarginal gyrus (BA 40; verbal), bilateral dorsolateral prefrontal cortex (BA 9/46; verbal and visual), and bilateral posterior superior parietal cortex (BA 7; verbal and visual). We defined the "verbal" masks as the peak cluster of voxels active in each of the verbal or verbal and visual regions for the words $>$ pictures contrast ( $p<0.001$; cluster size, 15 voxels). Similarly, we defined the "visual" masks as the peak voxels that were active in each of the visual or verbal and visual regions for the pictures $>$ words contrast ( $p<0.001$; cluster size, 15 voxels). We then calculated signal estimates within each ROI for task versus fixation baseline contrasts on the individual subject level. Next, we tested for correlations between these estimates and scores on the VVQ and WAIS. Using a Bonferroni correction for the number of regions of interest investigated for each condition (six), only two ROIs showed significant correlations with the task conditions of interest ( $p<0.05$, corrected). These regions were left SMG and right fusiform cortex (Fig. 2). The full pattern of results for these two regions is shown in Tables 2 and 3 and discussed in the following 

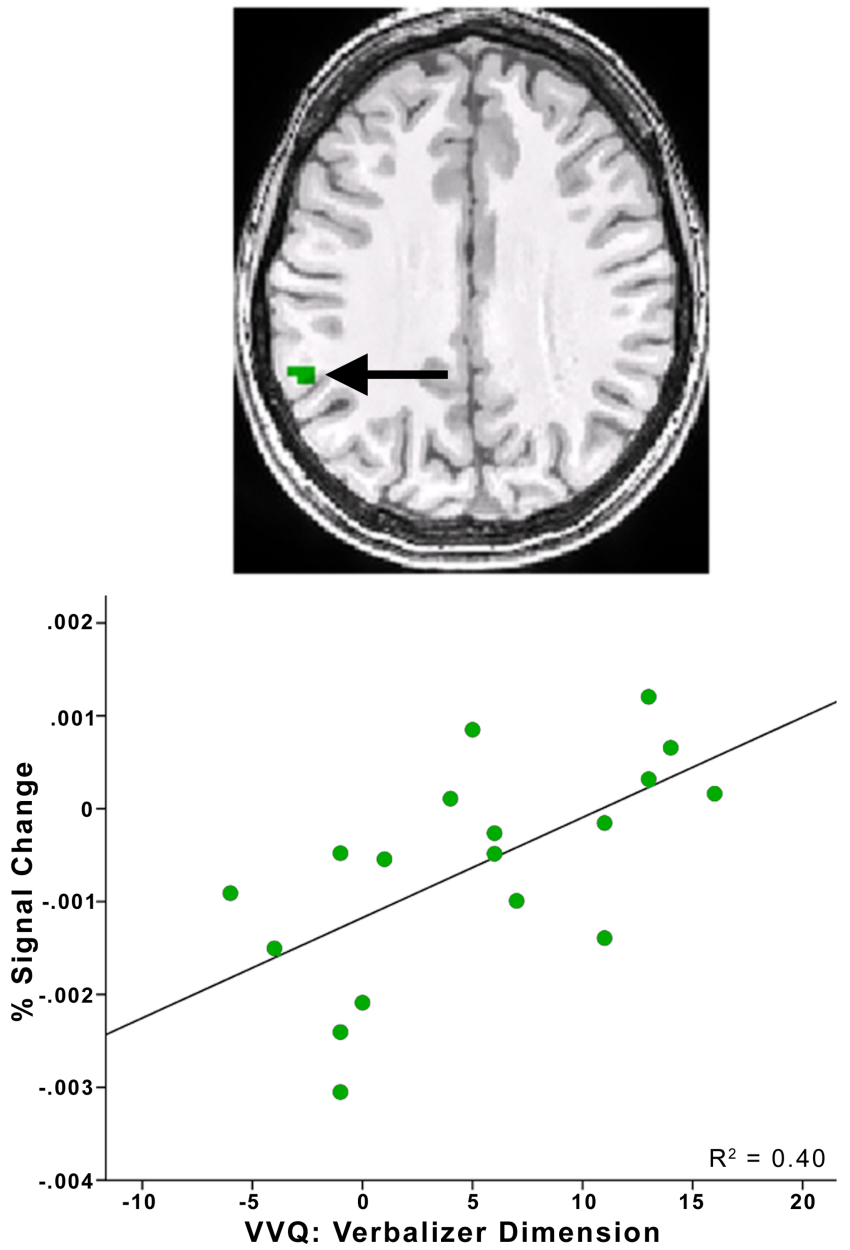
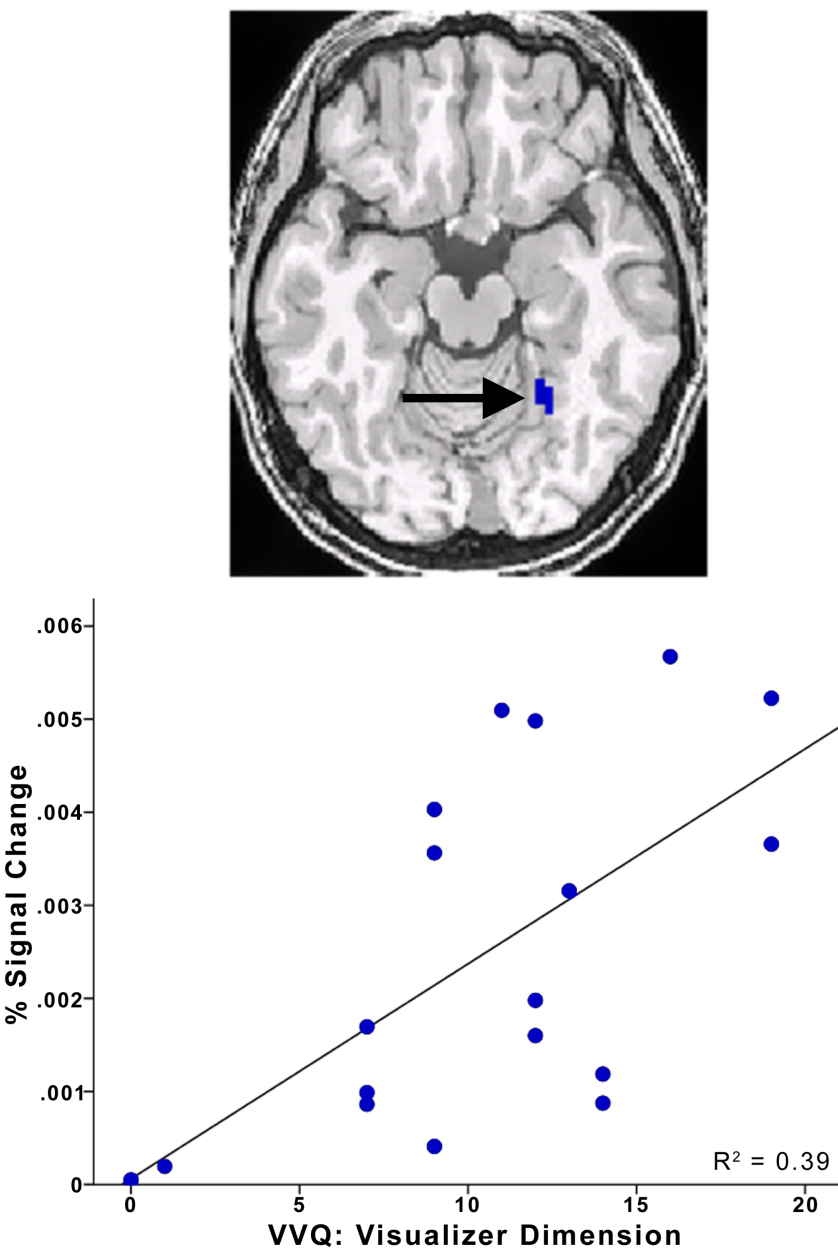

Figure 2. ROI analysis. Top, Shown in green is a section of the SMG ROI (left), defined as the voxels active in left SMG for the word-word $>$ picture-picture contrast at and above a threshold of $t_{(17)}>3.60, p<0.001$. Shown in blue is a section of the fusiform ROI (right), defined as the top 15 voxels active in right fusiform cortex for the picture-picture $>$ word-word contrast at the same statistical threshold as the SMG ROI. The top 15 voxels were chosen so that each ROI would comprise an equal number of voxels. Both the SMG and the fusiform ROI masks are superimposed onto axial slices of a representative subject's brain ( $Z=33$ and $Z=-15$, respectively). Bottom, Scatter plots depict the correlation between scores on the verbalizer dimension of the VVQ and activity in the SMG ROI during the picture-picture task (left) and between scores on the visualizer dimension of the VVQ and activity in the right fusiform ROI during the word-word task (right). Trend lines are plotted based on Pearson's correlations, and $R^{2}$ values are listed for each graph.

Table 3. Pearson's correlations ( $r$ ) between VVQ scores and right fusiform ROI activity by task

\begin{tabular}{llc}
\hline & VVQ: visualizer & VVQ: verbalizer \\
\hline Picture-picture condition & 0.12 & 0.07 \\
Word-word condition & $0.63^{* *}$ & 0.34 \\
Fixation condition & 0.36 & -0.17
\end{tabular}

${ }^{* *} p<0.01 ; p$ values calculated for two-tailed tests.

sections. The complete pattern of results including all ROIs is reported in the supplemental materials, available at www.jneurosci.org.

\section{Verbal style is correlated with increased SMG activity when viewing pictures}

Table 2 shows the results of a correlation analysis comparing scores on the separate dimensions of the VVQ with activity in the left SMG ROI during several task conditions (vs fixation baseline). As hypothesized, the only significant correlation for this ROI was between activity during the picture-picture condition and the scores on the verbalizer dimension of the VVQ $\left(r_{(17)}=\right.$ $0.63, p=0.005)$. In other words, the higher an individual scored on the verbalizer dimension, the more likely that individual was to show increased activity in SMG when presented with pictures comprising simple, nameable visual features (Fig. 2). As activity in SMG has previously been shown to correlate with phonological processing (Paulesu et al., 1993; Jonides et al., 1998; Smith and Jonides, 1998; Becker et al., 1999; Rypma and D’Esposito, 1999; Hickok et al., 2003; Postle, 2006), this finding is consistent with the hypothesis that when presented with pictures, those with a propensity toward the verbal style show a tendency to encode the stimuli in a verbal form.

As a further examination of the observed correlations, specifically to account for the possibility that the VVQ scores actually represent an ordinal rather than an interval scale (and therefore that the values reported as Pearson correlation coefficients are not accurate), Spearman correlation coefficients were also calculated. The outcome of all reported hypothesis tests (in this section and below) did not change as a result of these analyses.

Furthermore, as reported in Table 2, there was no significant correlation between verbalizer score and SMG activity during the word-word condition or during the fixation condition. This suggests that the significant correlation between verbalizer scores and SMG activity during the picture-picture condition was related to a cognitive process engaged during that task and not simply a general property of SMG activity for those who rate 
highly on the verbal cognitive style scale. To further test the specificity of this finding, we calculated a stepwise regression model of the verbalizer scores, as accounted for by SMG activity during the three relevant conditions (picture-picture, word-word, and fixation). After the fixation and word-word conditions were added to the model, adding the picture-picture condition still accounted for a significant portion of the variance in scores on the VVQ verbalizer dimension $(t=2.72, p=0.02)$. This result demonstrates that those who rated highly on the verbal style scale were preferentially recruiting SMG in a task-related manner. As the particular task that elicited this effect was the presentation of only pictures, the results suggest that those who are highly associated with the verbal style were converting the visual stimuli they encountered into a verbal code.

It is also of note that during fixation, SMG was somewhat more correlated with the verbalizer dimension than the visualizer dimension. This recruitment may be in the service of encoding features of their environment in the preferred verbal modality, even during nontask conditions. However, because the picturepicture condition had a stronger correlation with the verbalizer scale, and because it accounted for a significant portion of variance in verbalizer scores even after the other two conditions were modeled, these results serve as evidence that task-related differences in neural processing exist based on preference for the verbal or visual style, beyond any differences in baseline activity.

\section{Visualizers show increased activity in fusiform gyrus when reading words}

Table 3 shows the results of a correlation analysis comparing scores on the VVQ with activity in the right fusiform gyrus ROI for several task conditions (vs fixation baseline). Consistent with our hypothesis, the only significant correlation for this ROI was between activity during the word-word condition and the scores on the visualizer dimension of the VVQ $\left(r_{(17)}=0.63, p<0.01\right)$. In other words, the higher an individual scored on the visualizer dimension, the more likely they were to activate right fusiform cortex when presented with words that described visual features (Fig. 2, plot). As activity in this region has previously been shown to correlate with visual imagery of object features (Howard et al., 1998; Ishai et al., 2000; Ganis et al., 2004; Mechelli et al., 2004; Rich et al., 2006), this is consistent with the hypothesis that those who use the visual cognitive style engage in mental imagery of the named visual features.

Moreover, the lack of a significant relationship between visualizer score and right fusiform activity during the picture-picture or during the fixation condition (Table 3 ) suggests that the significant correlation between visualizer score and activity in the word-word condition was based on task-related fusiform activity. As with the SMG ROI analysis described above, to further examine the specificity of this finding, we calculated a stepwise regression model of how much variance in visualizer scores was accounted for by the activity in the right fusiform ROI during these three conditions. After the fixation and picture-picture conditions were added to the model, adding the word-word condition still accounted for a significant portion of the variance in visualizer scores $(t=2.89, p<0.012)$. This indicates that the observed correlation between visualizer score and fusiform activity was driven by activity during the word-word task and not simply a general property of activity in this region for those who scored highly on the visualizer dimension.

Although there was significant activity in both left and right fusiform gyri for the main effects contrast of pictures $>$ words, activity in the left fusiform ROI did not significantly correlate with either VVQ dimension during any task condition (picturesvisualizer: $r=-0.01, p=0.96$; pictures-verbalizer: $r=0.23, p=$ 0.37 ; words-visualizer, $r=0.16, p=0.52$; words-verbalizer, $r=$ $0.23, p=0.36)$. We hypothesized that fusiform activity would correlate with the visualizer dimension of the VVQ, but we did not have an a priori prediction about whether this activity would be localized to either hemisphere or whether it would be found bilaterally. Although some previous evidence does link right fusiform cortex activity to visual imagery and retrieval of colors and objects (Howard et al., 1998; Ganis et al., 2004; Rich et al., 2006; Vandenbulcke et al., 2006), future studies will be necessary to examine the exact nature of this pattern of hemispheric differences.

\section{Regional specificity of findings}

To explore whether the same correlations found in the SMG and fusiform ROIs would be observed in other brain regions, four whole-brain conjunction analyses were performed as follows. New models were fit for each group-level task-to-baseline comparison, separately modeling the scores from the two WAIS indices (POI, VCI) and the two VVQ dimensions (visualizer, verbalizer) as parametric regressors of brain activity for each subject at each voxel during the relevant task conditions (picture-picture for the verbalizer and VCI scores; word-word for the visualizer and POI scores). The resulting whole-brain parametric images were then combined with the pictures $>$ words contrast (for visualizer and POI parametric maps) or words $>$ pictures contrast (for verbalizer and VCI parametric maps), and the overlapping regions produced a new conjunction map for each analysis (four maps in total). Individual contrasts for these exploratory analyses were calculated at a threshold of $t>2.50, p<0.01$, resulting in a combined $t>6.25, p<0.0001$ for conjunction images (see figure in supplemental material, available at www. jneurosci.org). These conjunction maps represent the same relationships as the ROI analyses plotted in Figure 2 but do not have the same regional constraints. Very few cortical regions emerged from these analyses beyond the ones that were the focus of the ROI analyses. The only other cortical region in addition to left SMG to emerge from the conjunction of the parametric map of activity during the picture-picture condition modulated by verbalizer scores and the words $>$ pictures main effect contrast was an area of left anterior superior parietal lobe. Likewise, only one cortical region outside of right fusiform cortex was found as a result of combining the parametric map of activity during the word-word condition modulated by visualizer scores with the pictures $>$ words main effects contrast. This was an area near the left superior temporo-occipital junction. No regions were significantly active for the conjunction analyses that included the POI or VCI scores.

\section{Discussion}

In his monograph on the topic of human cognitive abilities, Carroll (1993) highlighted the importance of considering individual differences in various classes of domain-specific cognition. These abilities, he asserted, are separable from a more general intelligence factor and thus have unique influence on cognitive processing. In the present investigation, we present the first neuroimaging evidence exploring such individual differences in two types of modality-specific cognitive processing: visual and verbal cognitive styles.

We demonstrated here that those who were highly associated with the visual cognitive style recruit regions of visual cortex when processing written descriptions of visual features, propor- 
tionate to their self-reported inclination toward the visual cognitive style. Likewise, we also demonstrated that those who were highly associated with the verbal style recruit regions of phonologically responsive cortex when presented with easily nameable pictorial representations. The degree to which SMG was active during the picture condition was positively correlated with individuals' self-reported tendency toward the verbal cognitive style.

Thus, our analyses revealed two important findings with regard to our stated hypotheses. First, as the correlation between fusiform activity and visualizer score was found specifically during the word-word condition, this finding is consistent with the hypothesis that those who are associated with the visual style have a tendency to convert linguistically presented information into a visual mental representation. Second, as the link between the verbal cognitive style and activity in a phonological brain region was found only in the condition in which pictures were presented, this suggests that those who are associated with the verbal style have a tendency to convert pictorial information into linguistic representations.

The basic idea of cognitive styles, that different individuals process certain types of information differently, has appeared in many forms and has been part of many theories in various avenues of psychological research (Spearman, 1927; Guilford, 1967; Gardner, 1993; Mayer and Massa, 2003). Despite this widespread interest, however, a precise description of what constitutes a cognitive style, both from a behavioral and from a biological perspective, remains elusive. In examining the functional neural correlates of cognitive style during a set of relevant tasks, the present investigation takes a step toward characterizing these cognitive phenomena from a new perspective and thus is positioned to shed new light on the topic.

In particular, the present findings indicate some new aspects of what a cognitive style is. For example, the brain activity that was correlated with cognitive styles was found during the tasks that were presented in the nonpreferred modality (word-word for visual style and picture-picture for verbal style). This novel finding suggests that an important feature of processing in a specific cognitive style is that when one encounters a stimulus that is presented in a nonpreferred modality, one mentally converts that information into his or her preferred modality. The region of visual cortex in which activity was correlated with the visual style during the word-word condition was found across all subjects in this study to be more responsive to viewing the pictorial stimuli relative to viewing the words and has similarly been associated in other studies with perception and imagery of visual features, such as color (Howard et al., 1998; Ishai et al., 2000; Ganis et al., 2004; Mechelli et al., 2004; Rich et al., 2006). Similarly, the supramarginal gyrus, in which activity correlated with the verbal style during the picture-picture condition, was also active across all subjects for the contrast of word-word $>$ picture-picture, and has been implicated in many previous studies as a region that is involved in phonological processing and verbal working memory (Paulesu et al., 1993; Jonides et al., 1998; Smith and Jonides, 1998; Becker et al., 1999; Rypma and D'Esposito, 1999; Hickok et al., 2003; Postle, 2006). Thus, it is striking that, based on individual differences in cognitive style, these regions are recruited during the tasks for which they are not most strongly associated on the group level. This individualized activation is taken to be indicative of a strategy in which those who use the visual style mentally convert written information into a visual representation and those who use the verbal style convert visual information into a linguistic representation. Presumably, this converted representation facilitates processing and later recall. Future research can reveal more about the nature of these representations and the costs and consequences on task performance of engaging or not engaging in this conversion process.

One possible finding that could have reasonably been expected based on the extant neuroimaging literature was not observed in the present experiment. Specifically, one may have predicted that if the visual cognitive style was associated with more efficient processing of visual information, this style should correlate with less activity during the picture condition. A similar prediction might have been made for the verbal style and the word condition. The most straightforward explanation for why we do not find these negative correlations is that the present task was too easy to reliably drive gross activity changes in this manner, as these brain regions were activated by all participants to complete the task. In contrast, the effects we did observe that correlate with cognitive style are above and beyond the basic operations required to perceive and process the stimuli. Future studies may investigate whether a more difficult task would find this type of negative correlation between cognitive style and activity in brain regions that underlie the basic processes for that task.

As for the relationship between cognitive style and cognitive ability, although the present study did not find reliable correlations between VVQ scores and the subtests of the WAIS, it is important to note that this may be attributable to insufficient statistical power and is in contrast to previous research. In particular, at least one study using the same measure of cognitive style (Kirby et al., 1988) has shown that scores on the visualizer and verbalizer dimensions of the VVQ do correlate with objective measures of visuo-spatial and verbal abilities, respectively. Mayer and Massa (2003) also found that the original version of the VVQ correlated significantly with vocabulary test scores, as well as with the verbal section of the Scholastic Aptitude Test.

The findings from the present experiment, however, can be summarized by the description that cognitive style, operationalized here as scores on the VVQ, correlates with modality-specific neural activity in visual and verbal brain regions. What is still unanswered by the present research is whether major components of cognitive style are determined by factors that are linked to, or independent of, one's cognitive abilities. For instance, it may be the case, given proper training or motivation, that an individual can effectively learn to adopt a new cognitive style if doing so would facilitate problem solving in a specific domain. For example, a student who prefers the verbal style may be able to learn to visualize in certain situations where it would be helpful for a specific subject, such as organic chemistry. Future research on this topic may be able to suggest new and effective teaching methods that are tailored to the unique details of specific contexts and to the unique characteristics of specific individuals.

\section{References}

Annett M (1970) A classification of hand preference by association analysis. Br J Psychol 61:303-321.

Baddeley A (2003) Working memory: looking back and looking forward. Nat Rev Neurosci 4:829-839.

Becker JT, MacAndrew DK, Fiez JA (1999) A comment on the functional localization of the phonological storage subsystem of working memory. Brain Cogn 41:27-38.

Blajenkova O, Kozhevnikov M, Motes M (2006) Object-spatial imagery: a new self-report imagery questionnaire. Appl Cogn Psychol 20:239-263.

Boswell DL, Pickett JA (1991) A study of the internal consistency and factor structure of the Verbalizer-Visualizer questionnaire. J Mental Imagery 15:33-36.

Carroll JB (1993) Human cognitive abilities: a survey of factor-analytic studies. New York: Press Syndicate of the University of Cambridge. 
Cui X, Jeter CB, Yang D, Montague PR, Eagleman DM (2007) Vividness of mental imagery: individual variability can be measured objectively. Vis Res 47:474-478.

D’Esposito M (2007) From cognitive to neural models of working memory. Philos Trans R Soc Lond B Biol Sci. 362:761-772.

Friston KJ, Zarahn E, Josephs O, Henson RN, Dale AM (1999) Stochastic designs in event-related fMRI. Neuroimage 10:607-619.

Ganis G, Thompson WL, Kosslyn SM (2004) Brain areas underlying visual mental imagery and visual perception: an fMRI study. Brain Res Cogn Brain Res 20:226-241.

Gardner H (1993) Frames of mind, tenth anniversary edition. New York: Basic Books.

Goldberg RF, Perfetti CA, Schneider W (2006) Perceptual knowledge retrieval activates sensory brain regions. J Neurosci 26:4917-4921.

Green KE, Schroeder DH (1990) Psychometric quality of the verbalizervisualizer questionnaire as a measure of cognitive style. Psychol Rep 66:939-945.

Guilford JP (1967) The nature of human intelligence. New York: McGraw-Hill.

Halpern AR, Zatorre RJ (1999) When that tune runs through your head: a PET investigation of auditory imagery for familiar melodies. Cereb Cortex 9:697-704.

Hickok G, Buchsbaum B, Humphries C, Muftuler T (2003) Auditorymotor interaction revealed by fMRI: speech, music, and working memory in area spt. J Cogn Neurosci 15:673-682.

Howard RJ, ffytche DH, Barnes J, McKeefry D, Ha Y, Woodruff PW, Bullmore ET, Simmons A, Williams SC, David AS, Brammer M (1998) The functional anatomy of imagining and perceiving colour. Neuroreport 9:1019-1023.

Ishai A, Ungerleider LG, Haxby JV (2000) Distributed neural systems for the generation of visual images. Neuron 28:979-990.

Jonides J, Schumacher EH, Smith EE, Koeppe RA, Awh E, Reuter-Lorenz PA, Marshuetz C, Willis CR (1998) The role of parietal cortex in verbal working memory. J Neurosci 18:5026-5034.

Kirby JR, Moore PJ, Schofield NJ (1988) Verbal and visual learning styles. Contemp Educ Psychol 13:169-184.

Kosslyn SM, Thompson WL (2003) When is early visual cortex activated during visual mental imagery? Psychol Bull 129:723-746.

Kraemer DJ, Macrae CN, Green AE, Kelley WM (2005) Musical imagery: sound of silence activates auditory cortex. Nature 434:158.
Marks DF (1973) Visual imagery differences in the recall of pictures. Brit J Psychol 64:17-24.

Martin A (2007) The representation of object concepts in the brain. Annu Rev Psychol 58:25-45.

Mayer RE, Massa LJ (2003) Three facets of visual and verbal learners: cognitive ability, cognitive style, and learning preference. J Educ Psychol 95:833-846.

Mechelli A, Price CJ, Friston KJ, Ishai A (2004) Where bottom-up meets top-down: neuronal interactions during perception and imagery. Cereb Cortex 14:1256-1265.

Paulesu E, Frith CD, Frackowiak RS (1993) The neural correlates of the verbal component of working memory. Nature 362:342-345.

Postle BR (2006) Working memory as an emergent property of the mind and brain. Neuroscience 139:23-38.

Postle BR, Druzgal TJ, D’Esposito M (2003) Seeking the neural substrates of visual working memory storage. Cortex 39:927-946.

Ranganath C, D'Esposito M (2005) Directing the mind's eye: prefrontal, inferior and medial temporal mechanisms for visual working memory. Curr Opin Neurobiol 15:175-182.

Rich AN, Williams MA, Puce A, Syngeniotis A, Howard MA, McGlone F, Mattingley JB (2006) Neural correlates of imagined and synaesthetic colours. Neuropsychologia 44:2918-2925.

Richardson A (1977) Verbalizer-visualizer: a cognitive style dimension. J Mental Imagery 1:109-126.

Rypma B, D’Esposito M (1999) The roles of prefrontal brain regions in components of working memory: effects of memory load and individual differences. Proc Natl Acad Sci U S A 96:6558-6563.

Smith EE, Jonides J (1998) Neuroimaging analyses of human working memory. Proc Natl Acad Sci U S A 95:12061-12068.

Spearman C (1927) The abilities of man: their nature and measurement. New York: Macmillan.

Thompson-Schill SL (2003) Neuroimaging studies of semantic memory: inferring "how" from "where". Neuropsychologia 41:280-292.

Vandenbulcke M, Peeters R, Fannes K, Vandenberghe R (2006) Knowledge of visual attributes in the right hemisphere. Nat Neurosci 9:964-970.

Wager TD, Smith EE (2003) Neuroimaging studies of working memory: a meta-analysis. Cogn Affect Behav Neurosci 3:255-274.

Wechsler D (1997) Adult intelligence scale-3rd edition (WAIS-3R). San Antonio, TX: Harcourt Assessment. 